\title{
Bronchoalveolar and systemic cytokine profiles in patients with ARDS, severe pneumonia and cardiogenic pulmonary oedema
}

\author{
H. Schütte, J. Lohmeyer, S. Rosseau, S. Ziegler, C. Siebert, H. Kielisch, \\ H. Pralle, F. Grimminger, H. Morr, W. Seeger
}

Bronchoalveolar and systemic cytokine profiles in patients with ARDS, severe pneumonia and cardiogenic pulmonary oedema. H. Schütte, J. Lohmeyer, S. Rosseau, S. Ziegler, C. Siebert, H. Kielisch, H. Pralle, F. Grimminger, H. Morr, W. Seeger. CERS Journals Ltd 1996.

ABSTRACT: The aim of this study was to investigate whether bronchoalveolar lavage (BAL) and serum levels of proinflammatory cytokines discriminate between different entities of patients with acute respiratory failure.

BAL and circulating concentrations of interleukin-6 (IL-6), interleukin-8 (IL-8) and tumour necrosis factor- $\alpha(\mathrm{TNF}-\alpha)$ were measured in $\mathbf{7 4}$ mechanically-ventilated patients and 17 healthy controls. Patients were classified as cardiogenic pulmonary oedema (CPO), acute respiratory distress syndrome (ARDS), primary severe pneumonia (PN) and a combined group (PN+ARDS).

In all patients with ARDS and/or PN, markedly elevated BAL levels of IL-6 and IL-8 were detected, which were significantly greater than levels in CPO and healthy controls. Absolute quantities and time-course of these cytokines did not differentiate between the absence and presence of lung infection, or different categories of PN. Similarly, circulating IL-6 levels were comparably elevated in patients with ARDS and/or PN, whereas circulating IL-8 concentrations were inconsistently increased. TNF- $\alpha$ was rarely detected in BAL samples, but increased serum concentrations were measured in ARDS and/or PN patients.

Bronchoalveolar lavage levels of interleukin-6 and interleukin-8, but not tumour necrosis factor- $\alpha$, and serum concentrations of interleukin-6 are consistently elevated in acute respiratory distress syndrome and/or severe pneumonia, discriminating these entities from cardiogenic pulmonary oedema. Alveolar and systemic cytokine profiles do not differentiate between acute respiratory distress syndrome in the absence of lung infection and states of severe primary or secondary pneumonia, which evidently present with comparable local and systemic inflammatory sequelae.

Eur Respir J., 1996, 9, 1858-1867.

Cytokines are involved in a variety of inflammatory lung diseases, but their pathogenetic role and their significance as diagnostic tools are still controversial. Interleukin-8 (IL-8) is important for the recruitment of inflammatory cells into the alveolar space, which is a hallmark of the acute respiratory distress syndrome (ARDS) and severe pneumonia $(\mathrm{PN})$. Elevated levels of IL-8 have been shown in bronchoalveolar lavage (BAL) fluids of ARDS patients [1-4], as well as under general conditions of sepsis and multiorgan failure [5-7]. Tumour necrosis factor- $\alpha$ (TNF- $\alpha)$ is secreted by monocytes or macrophages, including those of the alveolar space, after exposure to bacterial lipopolysaccharides and a variety of proinflammatory mediators $[8,9]$. Together with other cytokines, it appears to orchestrate the response to an inflammatory stimulus. Many features of septic shock [10-13] and ARDS $[14,15]$ have been ascribed to TNF$\alpha$, but measurements of this cytokine under such clinical conditions (serum and lavage fluid) have produced inconsistent results [16-24]. Interleukin-6 (IL-6) is produced by a variety of inflammatory cells in response to other cytokines or bacterial agents. Circulating IL-6 was found to be an important marker and mediator of sys-
Dept of Internal Medicine, Justus-Liebig University, Klinikstrasse, Giessen, FRG.

Correspondence: W. Seeger

Dept of Internal Medicine

Justus-Liebig University

Klinikstrasse 36

35385 Giessen

FRG

Keywords: Acute respiratory distress syndrome

bronchoalveolar lavage

interleukin-5

interleukin-8

tumour necrosis factor- $\alpha$

Received: July 61995

Accepted after revision April 91996

Parts of the doctoral theses of S.Z. and C.S. are incorporated in this report. temic inflammatory responses ("alarm hormone"), particularly in sepsis $[5,17,22,25,26]$, whereas only a few studies have focused on the appearance of this cytokine in the alveolar space [27-30].

In the present study, the incidence of IL- 6 , IL- 8 and TNF- $\alpha$ were investigated in the alveolar and vascular compartments of a total of 74 patients requiring mechanical ventilation due to acute respiratory failure. The data were compared to healthy controls and to an additional "control" group of patients with cardiogenic pulmonary oedema, in an attempt to differentiate between the primary sequelae of inflammatory lung injury and secondary consequences of severe functional impairment of gas exchange necessitating respirator therapy. Moreover, we focused particularly on primary and secondary pulmonary infections as contributors to lung cytokine generation: despite a recent "uniforming" definition of ARDS [31], the patients were divided into those with ARDS in the absence of lung infection, those with primary severe PN and those with features of ARDS and PN. In addition, we sought possible differences in lung and systemic cytokine profiles among different categories of PN. 


\section{Methods}

Patients included in the study were recruited from the intensive care unit of the Department of Internal Medicine, JLU-University Giessen. The project was approved by the Ethics Committee of the Justus-Liebig-University, and informed consent was obtained either from the patient or closest relatives. All patients were examined by BAL for clinical purposes; the large majority of them within $72 \mathrm{~h}$ after intubation. In selected patients - depending on the clinical course - bronchoscopy and BAL were repeated at later time-points. All patients were mechanically-ventilated; inspiratory oxygen fraction $\left(F \mathrm{I}, \mathrm{O}_{2}\right)$ and respirator settings including positive end-expiratory pressure (PEEP) were chosen according to the requirements of pulmonary insufficiency. General therapeutic approaches included parenteral nutrition, volume substitution and antibiotic drugs. Vasoactive or inotropic drugs were administered according to the patients history, and haemodynamic variables as determined by right heart catheterization.

\section{Definitions of categories}

Each patient was classified in one of the four main categories described below; there was no overlap and no change of patients between these main categories. Healthy volunteers $(n=17)$ without a history of cardiac or pulmonary disease served as the control group. The distribution of age, body weight and sex in patient groups and controls displayed negligible variations. General exclusion criteria for entry into this study were lung cancer, chronic obstructive or interstitial lung disease, and head trauma, stroke or subarachnoidal haemorrhage.

Severe pneumonia $(P N)$. In total, 38 patients with a clinical history of primary lung infection were examined (mean age 57 yrs; 31 males and 7 females). Diagnostic criteria were fever, tachycardia, dyspnoea, typical auscultatory findings, characteristic chest roentgenograms and microbiological identification of pathogens in the lower respiratory tract (bronchoscopy; pathogens listed in table 1). The pulmonary capillary wedge pressure ranged $<16 \mathrm{mmHg}$, and there was no history of acute or chronic left heart failure. In this group of patients, based on radiological criteria according to FRASER et al. [32], subgroups of: bronchopneumonia (patchy peribronchially centred consolidation, $\mathrm{n}=9$ ); alveolar PN (homogeneous, sharply demarcated consolidations with air bronchogram, $\mathrm{n}=14$ ); and aspiration PN (extensive bilateral airspace consolidation, widespread acinar shadows, irregular opacities, with a history of aspiration of gastric contents, n=11) were analysed. Four patients could not be ascribed to a subgroup.

Acute respiratory distress syndrome $(A R D S)$ in the absence of primary lung infection. In total, 12 patients were examined (mean age 51 yrs; 10 males and 2 females). For diagnosis of ARDS, the following general criteria were required: 1) An initial, typical underlying catastrophic event. The predominant event in this group was sepsis $(n=7)$; other causes included shock $(n=3)$, aspiration of gastric contents with Mendelson's syndrome $(n=1)$, and pancreatitis $(n=1) ; 2)$ Roentgenographic diffuse and bila-
Table 1. - Specific microorganisms detected in the respiratory tract in patients with pneumonia and those with pneumonia and ARDS

\begin{tabular}{lcc}
\hline Pathogen & PN & PN + ARDS \\
& $\mathrm{n}$ & $\mathrm{n}$ \\
\hline Pseudomonas spp. & 6 & 7 \\
Haemophilus influenzae & 2 & 1 \\
Klebsiella spp. & 5 & 7 \\
Escherichia coli & 1 & - \\
Streptococcus pneumoniae & 8 & 2 \\
Other Streptococcus spp. & 7 & 1 \\
Staphylococcus aureus & 3 & 3 \\
Coagulase(-) Staphylococcus & 9 & 2 \\
Anaerobic pathogens & 3 & - \\
Other Gram(-) pathogens & 2 & 3 \\
Other Gram(+) pathogens & - & - \\
Candida spp. & 12 & 14 \\
Aspergillus spp. & 2 & - \\
Other fungal pathogens & - & - \\
\hline
\end{tabular}

PN: pneumonia; ARDS: acute respiratory distress syndrome.

teral alveolar infiltrates; 3) Pulmonary capillary wedge pressure $<16 \mathrm{mmHg}$ and absence of acute or chronic left heart failure; 4) Absence of microorganisms upon first bronchoscopy.

$A R D S$ in combination with pneumonia $(P N+A R D S)$. In total, 18 patients (mean age 46 yrs; 14 males and 4 females) were separated into two subcategories. The first subgroup ( $\mathrm{PN} \rightarrow$ ARDS) was formed by patients with the initial clinical diagnosis of "severe PN" with circumscript lung infiltrates, in whom sequential chest radiographs displayed rapid, diffuse bilateral spreading of infiltrates, imposing a typical radiographic pattern of ARDS $(n=9)$. The second group (ARDS $\rightarrow$ PN) consisted of patients initially classified as "ARDS" with underlying events differing from PN, who acquired secondary (nosocomial) PN within $<72 \mathrm{~h}(\mathrm{n}=9)$; underlying causes were sepsis $(n=4)$, aspiration $(n=4)$, and pancreatitis $(n=1)$. PN was always confirmed by microbiological identification of pathogenic microorganisms in BAL fluid.

Cardiogenic pulmonary oedema $(\mathrm{CPO})$. Six patients requiring mechanical ventilation due to cardiogenic pulmonary oedema were enrolled in the study; none of them met the criteria for ARDS or PN. All of these patients had clinical and radiographic signs of severe pulmonary congestion and alveolar flooding due to left-sided heart failure, and all of them had pulmonary capillary wedge pressures greater than $18 \mathrm{mmHg}$. All patients suffered from ischaemic heart disease. Owing to clinical improvement under therapy, mechanical ventilation in this patient group was never required for more than $48 \mathrm{~h}$.

For direct comparison of ARDS and PN patients with the cardiogenic oedema group, all patients with ARDS in the absence or presence of secondary PN were regarded as "primary ARDS", and all patients with PN with and without secondary ("ARDS-like") bilateral diffuse spreading of infiltrates were regarded as "primary PN".

\section{Bronchoalveolar lavage}

Flexible fibreoptic bronchoscopy with BAL was performed as a routine diagnostic procedure. Ten $20 \mathrm{~mL}$ aliquots of sterile saline were infused into one segment 
of the lingula or the right middle lobe and removed by gentle suction (recovery 55-70\%). In patients with severe $\mathrm{PN}$, the lung with predominant infection was examined. Lavage fluids were filtered through sterile gauze, collected on ice and immediately centrifuged at $200 \times \mathrm{g}$ for $10 \mathrm{~min}$. Supernatant aliquots were frozen in liquid nitrogen and stored at $-85^{\circ} \mathrm{C}$ for subsequent measurements. Directly before starting the BAL procedure, samples of central venous blood were collected, allowed to clot for $30 \mathrm{~min}$ and centrifuged at $1,500 \times \mathrm{g}$ for $15 \mathrm{~min}$. The serum was decanted and stored at $\left(-85^{\circ} \mathrm{C}\right)$. Endotoxin-free plastic materials for collection, centrifugation and storage of serum and BAL samples were used throughout to avoid secondary cytokine production due to endotoxin contamination [21]. For quantification of alveolar lining fluid, urea was used as an endogenous marker of dilution [33]. Blood gas analysis was performed in each patient within 30 min before the BAL procedure.

\section{Cytokine assays}

IL- 6 and IL-8 in serum and BAL fluids were measured by commercially available assays (IL-6 enzyme-amplified sensitivity immunoassay (EASIA) and IL-8 enzyme-linked immunosorbent assay (ELISA); Medgenix Diagnostics, Ratingen, Germany). The minimum detectable concentrations were $3 \mathrm{pg} \cdot \mathrm{mL}^{-1}$ of IL- 6 and $11 \mathrm{pg} \cdot \mathrm{mL}^{-1}$ of IL-8, respectively. TNF- $\alpha$ in BAL fluids was measured by means of three assay systems. Bioactivity of TNF- $\alpha$ in BAL samples was determined in a cytolytic cell assay in the mouse fibrosarcoma cell line WEHI 164 clone 13 (kindly donated by T. Espevik, Institute of Cancer Research, University of Trondheim, Norway), as described previously [34]. The WEHI cells $\left(2 \times 10^{4}\right)$ were incubated with serial dilutions of BAL fluids in microtitre wells (Nunc). After $18 \mathrm{~h}$, dimethylthiazolyl-diphenyltetrazolium-bromide (MTT; $5 \mathrm{mg} \cdot \mathrm{mL}^{-1}$ in phosphatebuffered saline (PBS); $100 \mu \mathrm{L} \cdot$ well $^{-1}$ ) was added. The reaction was stopped after $4 \mathrm{~h}$ by addition of $5 \%$ formic acid in 2-propanol, and the content of reduced MTT was read in a micro-ELISA autoreader $(570 \mathrm{~nm})$. The titre of TNF- $\alpha$ is expressed in units. $\mathrm{mL}^{-1}$ and was chosen as the reciprocal of the dilution necessary to cause $50 \%$ cytotoxi-city. Recombinant human TNF- $\alpha$ (Genzyme,
Rüsselsheim, Germany) served as standard in all assays. The sensitivity of WEHI 164.13 ranged $1-3$ pg protein.cytolytic unit ${ }^{-1}$ in the different tests. Antigenic TNF$\alpha$ in BAL fluids was determined by two ELISAs (Medgenix Diagnostics, Ra-tingen; and Genzyme, Rüsselsheim, Germany; detection limits $3 \mathrm{pg} \cdot \mathrm{mL}^{-1}$ each). Recovery of TNF- $\alpha$ in BAL fluids, as determined by addition of recombinant human TNF- $\alpha$ (Genzyme, Rüsselsheim, Germany) to normal, freshly obtained lavage fluids, ranged 96-107\%, as measured by the Genzyme ELISA ( $n=10$ experiments; with BAL fluids from ARDS and PN patients). TNF- $\alpha$ analysis in serum samples was also performed by means of the Genzyme ELISA kit.

\section{Data analysis}

Differences in cytokine concentrations among patient groups were analysed by the Kruskal-Wallis H-Test and the Wilcoxon-Mann-Whitney-Test. Correlations between parameters were analysed by calculating the Spearman Rank Correlation Coefficient. P-values of less than 0.05 were considered to represent a significant difference or correlation. For interpretation of multiple comparisons, the Bonferroni correction was considered. All statistical procedures were performed with the Statistical Package for the Social Sciences (SPSS) ${ }^{\mathrm{TM}}$ for MS Windows ${ }^{\mathrm{TM}}$ analysis system. "Survival" of patients was defined as being alive 4 weeks after onset of mechanical ventilation.

\section{Results}

\section{Basic data}

All four groups of patients (ARDS, PN, PN + ARDS, $\mathrm{CPO})$ displayed a comparable degree of respiratory failure, as reflected by arterial oxygen tension $\left(\mathrm{Pa}, \mathrm{O}_{2}\right) / F \mathrm{I}, \mathrm{O}_{2}$ ratios (table 2). Polymorphonuclear neutrophils (PMNs) were markedly elevated in BAL fluids of all patients with ARDS and/or PN ( $\mathrm{p}<0.001$ compared to controls), whereas patients with cardiogenic pulmonary oedema displayed normal levels of PMNs. In all four groups, BAL protein concentrations and serum $\mathrm{C}$-reactive protein (CRP) levels were significantly higher than in controls

Table 2. - Selected basic variables of patients with: pneumonia (PN); acute respiratory distress syndrome (ARDS); combined forms (PN+ARDS); cardiogenic pulmonary oedema; and healthy controls

\begin{tabular}{|c|c|c|c|c|c|}
\hline & Pneumonia & ARDS & $\mathrm{PN}+\mathrm{ARDS}$ & $\begin{array}{l}\text { Cardiogenic } \\
\text { oedema }\end{array}$ & Control \\
\hline Protein in BAL $\mu \mathrm{g} \cdot \mathrm{mL}^{-1}$ & $\begin{array}{l}525 \pm 129 \dagger \\
(313)\end{array}$ & $\begin{array}{l}671 \pm 256^{\dagger} \\
(174)\end{array}$ & $\begin{array}{c}437 \pm 66^{\dagger} \\
(257)\end{array}$ & $\begin{array}{c}291 \pm 81^{\dagger} \\
(207)\end{array}$ & $\begin{array}{c}73 \pm 13.5 \\
(49.7)\end{array}$ \\
\hline $\mathrm{PMN}$ in BAL \% & $\begin{array}{l}36.2 \pm 4.4 \dagger \\
(26)\end{array}$ & $\begin{array}{c}42.5 \pm 7.5^{\dagger} \\
(44)\end{array}$ & $\begin{array}{l}32.7 \pm 6.5^{\dagger} \\
(14)\end{array}$ & $5.5 \pm 1.1$ & $\begin{array}{l}3.3 \pm 1.26 \\
(2.5)\end{array}$ \\
\hline $\begin{array}{l}\mathrm{PMN} \text { counts in BAL } \\
10^{6} \cdot \mathrm{mL}^{-1}\end{array}$ & $\begin{array}{c}22.3 \pm 11.1^{\dagger} \\
(4.19)\end{array}$ & $\begin{array}{c}16.1 \pm 5.8^{\dagger} \\
(4.49)\end{array}$ & $\begin{array}{l}5.7 \pm 1.2^{\dagger} \\
(2.78)\end{array}$ & $\begin{array}{l}0.4 \pm 0.11 \\
(0.37)\end{array}$ & $\begin{array}{l}0.32 \pm 0.09 \\
\quad(0.28)\end{array}$ \\
\hline Serum CRP $\mathrm{mg} \cdot \mathrm{L}^{-1}$ & $\begin{array}{c}159 \pm 15^{\dagger} \\
(141)\end{array}$ & $\begin{array}{c}235 \pm 33^{\dagger} \\
(222)^{\dagger}\end{array}$ & $\begin{array}{c}230 \pm 28^{\dagger} \\
(173)\end{array}$ & $\begin{array}{c}93 \pm 25^{\dagger} \\
(69)\end{array}$ & $<5$ \\
\hline 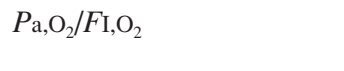 & $\begin{array}{c}204 \pm 17 \\
(171)\end{array}$ & $\begin{array}{c}213 \pm 21 \\
(235)\end{array}$ & $\begin{array}{r}196 \pm 15 \\
(186)\end{array}$ & $\begin{array}{c}226 \pm 20 \\
(243)\end{array}$ & \\
\hline
\end{tabular}

Values are presented as mean \pm SEM and median in brackets for each group. BAL protein values are presented as directly measured concentrations without urea-correction for dilution. All patients with ARDS and/or PN and cardiogenic oedema displayed elevated BAL protein concentrations and serum CRP levels ( $\dagger$ : $p<0.001$ each, compared to controls). Significant differences between these groups were not observed for these two variables or for the $\mathrm{Pa}, \mathrm{O}_{2} / F \mathrm{I}, \mathrm{O}_{2}$ ratios. BAL neutrophils were significantly elevated in all ARDS/PN patients ( ${ }^{\circ}: \mathrm{p}<0.001$ each, compared to controls), but not in patients with cardiogenic pulmonary oedema. BAL: bronchoalveolar lavage; PMN: polymorphonuclear neutrophils; CRP: C-reactive protein; $\mathrm{Pa}_{\mathrm{a}} \mathrm{O}_{2}$ : arterial oxygen tension in $\mathrm{mmHg}$; $\mathrm{F}_{\mathrm{I}, \mathrm{O}_{2}}$ : inspiratory oxygen fraction. 


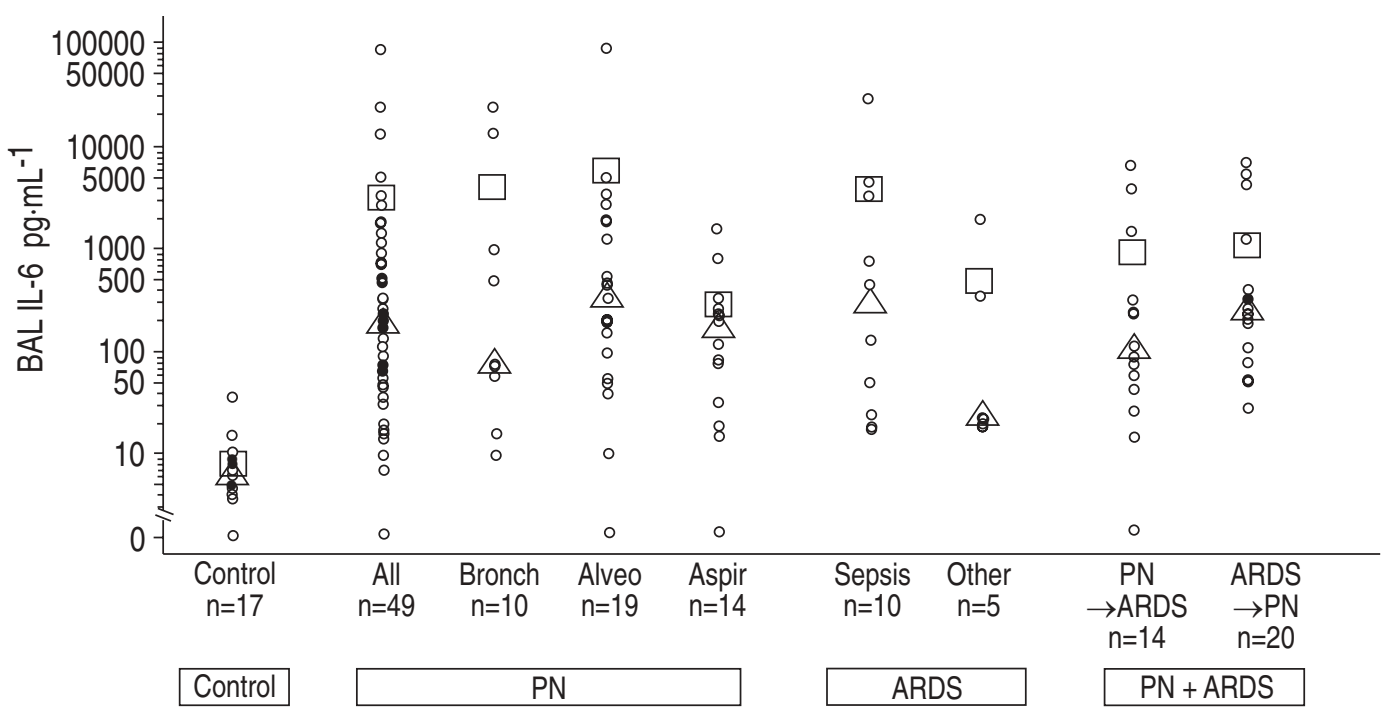

Fig. 1. - BAL IL-6 values in patients with pneumonia (PN), acute respiratory distress syndrome (ARDS), combined forms (PN+ARDS) and controls. All values are presented as directly measured concentrations without urea-correction for dilution. Open circles $(O)$ represent single measurements; additionally, mean $(\square)$ and median $(\Delta)$ of each subgroup are given. PN, ARDS and PN+ARDS were all significantly different from controls $(\mathrm{p}<0.0001)$. No differences were observed between these main groups and between subgroups of either main group. Bronch: bronchopneumonia; Alveo: alveolar pneumonia; Aspir: aspiration pneumonia; PN $\rightarrow$ ARDS: patients initially classified as "severe PN", in whom ARDS developed; ARDS $\rightarrow$ PN: patients initially classified as "ARDS", who acquired secondary PN.

$(\mathrm{p}<0.001)$ (table 2); values in patients with cardiogenic oedema ranged below those in ARDS and PN patients.

\section{IL-6 in BAL and serum}

Highly elevated concentrations of IL-6 were noted in BAL fluids of patients with ARDS and/or PN, as compared to control patients (fig. 1). This was true for all subgroups, namely, bronchopneumonia, alveolar and aspiration pneumonia, septic and nonseptic ARDS, ARDS with nosocomial pneumonia and pneumonia with secondary diffuse spreading of infiltrates (PN $\rightarrow$ ARDS). No statistical difference was noted among the various subgroups. BAL IL-6 levels remained elevated for more than 10 days after intubation in the groups with ARDS and PN, and then significantly decreased in the latter group (fig. 2). In contrast, BAL IL-6 concentrations of patients with cardiogenic pulmonary oedema were only slightly elevated as compared to controls, and data ranged significantly below those of ARDS and PN patients. Serum IL-6 levels were also markedly elevated in all three ARDS/PN groups and subgroups (fig. 3). Again, no statistical difference among the various subgroups was noted.

\section{IL-8 in BAL and serum}

All patients with ARDS and/or PN displayed highly significantly elevated BAL levels of IL-8. As detailed in figure 4, this again was true for all subgroups: no statistical difference was noted between or within the three groups of ARDS and PN. In addition, the kinetics of BAL IL-8 levels were nearly superimposable for patients with primary ARDS and those with primary PN (fig 5). In analogy with IL-6, marked elevation of the BAL concentrations of this cytokine was observed for more than 10 days. In contrast, BAL fluids obtained from patients with cardiogenic pulmonary oedema displayed only slightly elevated IL-8 levels, which significantly differed from the data in the ARDS and PN groups. In serum

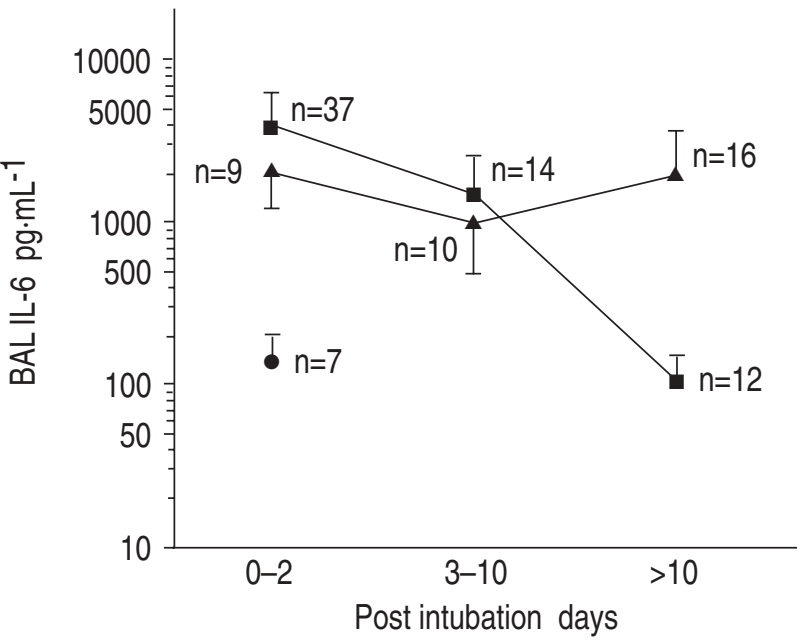

Fig. 2. - Time course of BAL IL-6 levels in patients with primary acute respiratory distress syndrome (ARDS), and primary pneumonia and initial value for cardiogenic oedema. For different times after intubation, values are depicted as mean \pm SEM of directly measured concentrations without urea-correction. Numbers next to symbols indicate the number of samples. BAL IL- 6 values in patients with cardiogenic oedema (only obtained within the first $48 \mathrm{~h}$ ) were significantly lower than in patients with ARDS and with pneumonia $(\mathrm{p}<0.05)$, but higher than in controls ( $\mathrm{p}<0.01$, see fig 1 for control values). The ARDS and pneumonia groups did not differ significantly. Within the pneumonia group, the later data ( $>10$ days) were significantly lower than the early data $(0-2$ days; $\mathrm{p}<0.01)$. $\_$: primary ARDS; — $\longrightarrow$ : primary pneumonia; —- : cardiogenic oedema. BAL: bronchoalveolar lavage; IL-6: interleukin-6.

samples taken in parallel with the BAL fluids, a broad scattering of data was noted. However, a significant elevation in comparison with the control group was only found for the patients suffering from PN + ARDS (fig. 6).

\section{$T N F-\alpha$ in $B A L$ and serum}

TNF- $\alpha$ bioactivity was not detected in any BAL fluid, either in PN, ARDS, or cardiogenic pulmonary oedema. 


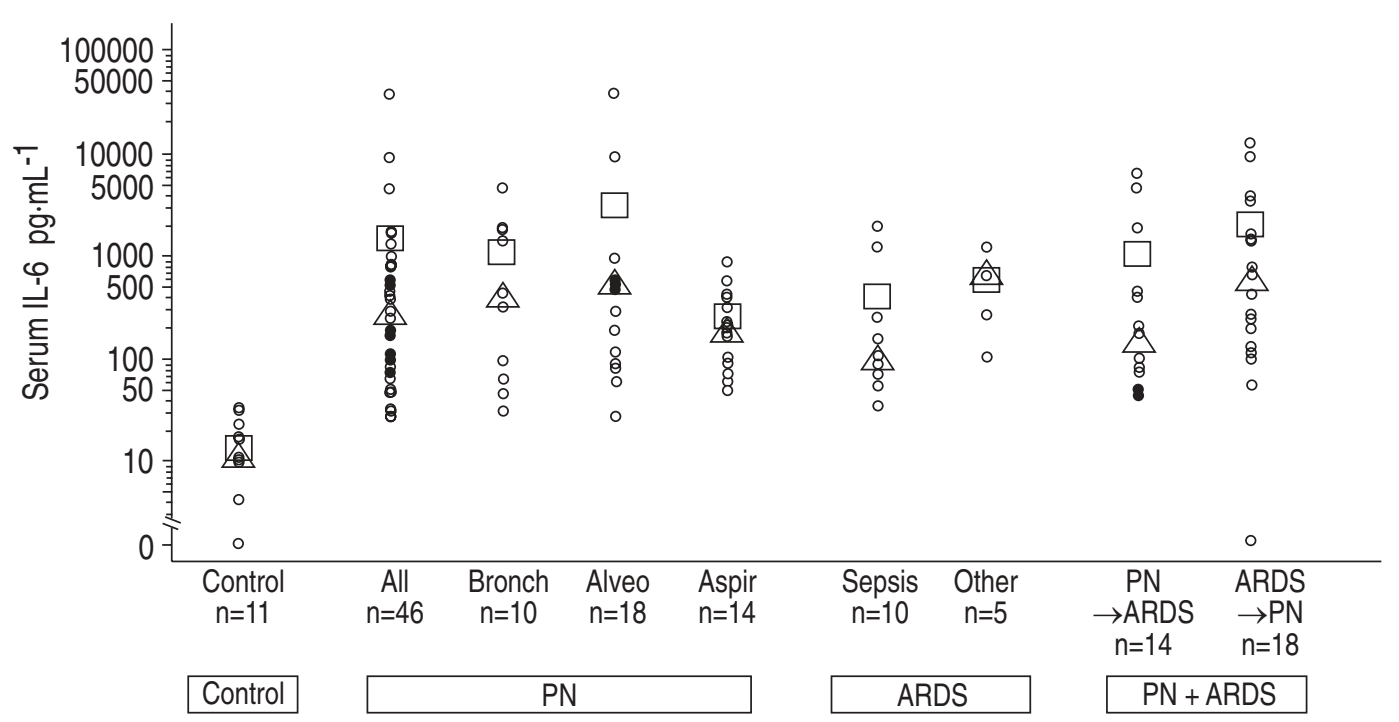

Fig. 3. - Serum IL-6 values in patients with pneumonia (PN), acute respiratory distress syndrome (ARDS), combined forms (PN+ARDS) and controls. Open circles $(O)$ represent single measurements; additionally, mean $(\square)$ and median $(\Delta)$ of each subgroup are given. PN, ARDS and PN+ARDS were all significantly different from controls $(\mathrm{p}<0.0001)$. No differences were observed between these main groups and between subgroups of either main group. For definitions see legend to figure 1.

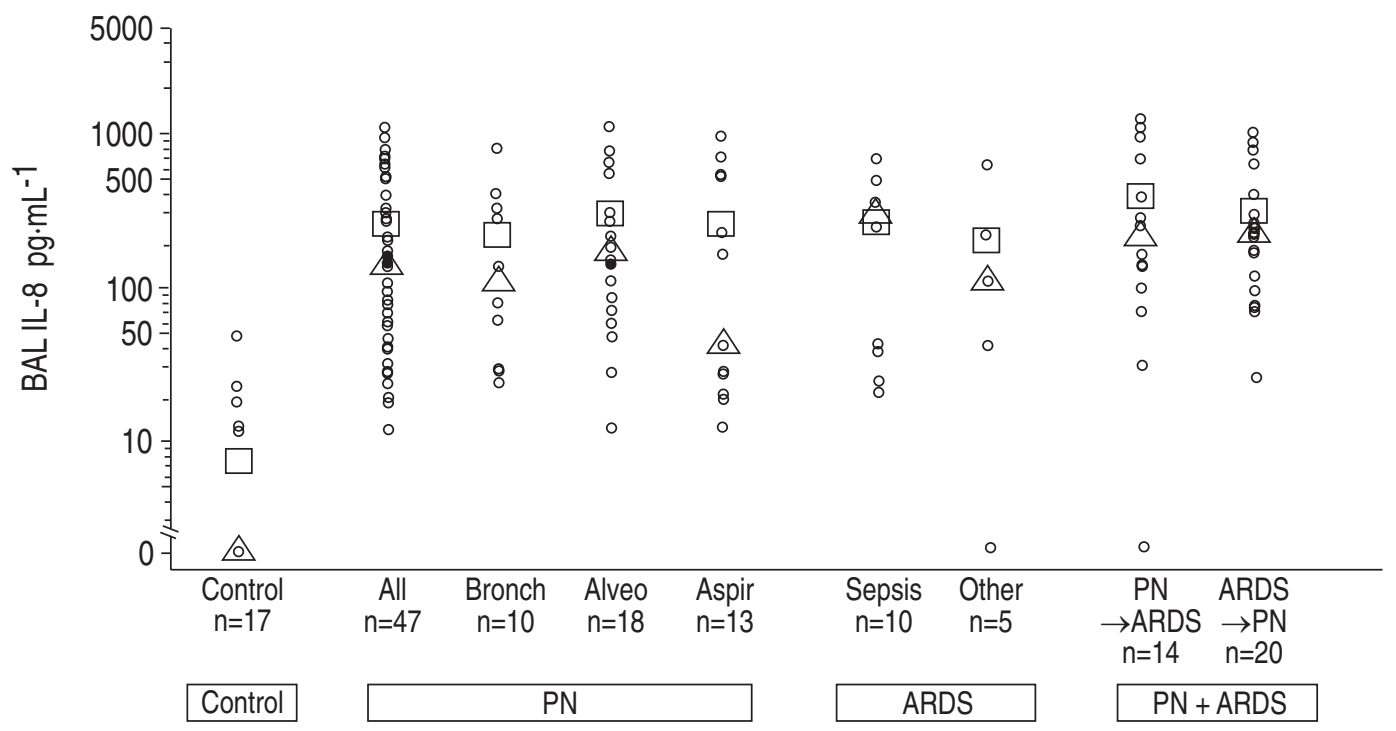

Fig. 4. - BAL IL-8 values in patients with pneumonia (PN), acute respiratory distress syndrome (ARDS), combined forms (PN+ARDS) and controls. All values are presented as directly measured concentrations without urea-correction for dilution. Open circles $(O)$ represent single measurements; additionally, mean $(\square)$ and median $(\Delta)$ of each subgroup are given. PN, ARDS and PN+ARDS were all significantly different from controls $(\mathrm{p}<0.0001)$. No differences were observed between these main groups and between subgroups of either main group. IL-8: interleukin-8. For further definitions see legend to figure 1 .

Moreover, in additional measurements with two ELISA techniques, antigenic TNF- $\alpha$ was very rarely measured in the BAL samples of these patients (only $4 \mathrm{PN} \pm \mathrm{ARDS}$ patients, range $66-384 \mathrm{pg} \cdot \mathrm{mL}^{-1}$; no ARDS patient). In contrast, circulating antigenic TNF- $\alpha$ levels were significantly elevated in all populations with ARDS, PN or PN + ARDS, as compared to controls (fig. 7). Main group and subgroup analysis of these values revealed no significant differences.

\section{Alveolar lining fluid}

Correction of single BAL data according to the urea method in order to obtain concentrations in the assumed volume of alveolar lining fluid resulted in $\approx 10$ fold (mean factor; range 2.5-83 fold) higher cytokine concentrations. Both urea-corrected IL-6 and IL-8 values were substantially higher than the corresponding serum levels in either patient group $(\mathrm{p} \leq 0.045)$, indicating local production of these cytokines. However, statistical analysis of these urea-corrected data did not reveal any previously unobserved differences among the various groups and subgroups of patients.

\section{Survival}

Survival rates were $42 \%$ for ARDS, $58 \%$ for PN and $61 \%$ for PN+ARDS patients, respectively. Due to the 
scattering of data, a broad overlap of cytokine data was noted between surviving and nonsurviving patients, and no significant difference was observed except for serum IL-8 values in PN patients, which were higher in nonsurviving than in surviving patients (table 3 ). All but one patient with cardiogenic oedema survived.

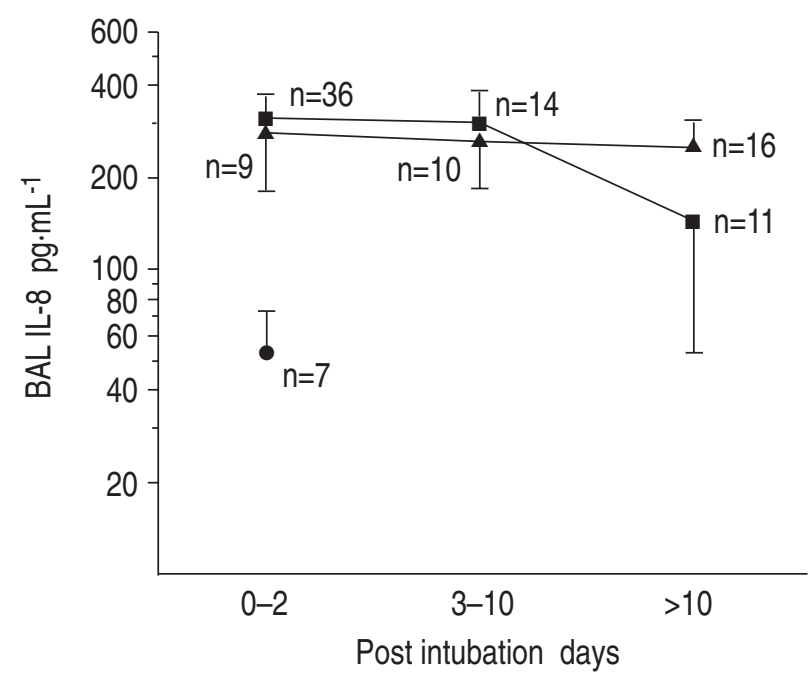

Fig. 5. - Time course of BAL IL-8 levels in patients with primary acute respiratory distress syndrome (ARDS), and primary pneumonia and initial value for cardiogenic oedema. For different times after intubation, values are depicted as mean \pm SEM of directly measured concentrations without urea-correction. Numbers next to symbols indicate the number of samples. BAL IL-8 values in patients with cardiogenic oedema (only obtained within the first $48 \mathrm{~h}$ ) were significantly lower than in patients with ARDS and with pneumonia patients $(\mathrm{p}<0.01)$, but higher than in controls $(\mathrm{p}<0.001$, see fig 4 for control values). The ARDS and pneumonia groups did not differ significantly. Within the pneumonia group, the later data ( $>10$ days) were significantly lower than the early data $(0-2$ and $2-10$ days; $\mathrm{p}<0.05)$. _ $\quad$ : primary ARDS; $\longrightarrow$ : primary pneumonia; $\longrightarrow$ : cardiogenic oedema.

\section{Correlations}

In patients with ARDS, PN or PN + ARDS, both BAL IL-6 and BAL IL-8 levels diplayed positive correlations with other variables of lung inflammation, in particular alveolar protein load and neutrophil influx; significance levels are presented in table 4. Overall, such correlation was of limited strength (highest correlation coefficient 0.69 for IL-8 and BAL protein in ARDS) and significance was not achieved in all cases. BAL IL- 8 and BAL IL-6 levels were significantly correlated with each other (table 5); and serum IL-6 levels displayed significant correlation with serum CRP values (table 4) and BAL IL-6 concentrations (table 5) in PN and PN+ARDS groups only.

\section{Discussion}

The present study investigated serum and alveolar space cytokine levels in 74 patients, requiring mechanical ventilation due to acute respiratory insufficiency over a period of 2 yrs, as compared to 17 healthy controls. In particular, differences between cardiogenic pulmonary oedema and states of inflammatory lung injury were sought, and the impact of primary or secondary lung infection on pulmonary cytokine generation. In essence, markedly elevated BAL levels of IL-6 and IL-8 were detected in virtually all individual patients with ARDS and severe pneumonia, clearly separating these entities from cardiogenic pulmonary oedema. However, the alveolar or serum cytokine profiles did not discriminate between absence or presence of primary or secondary lung infection and among subgroups of pneumonia.

\section{Classification of patients}

According to the American-European Consensus Conference on ARDS [31], recommended criteria for acute

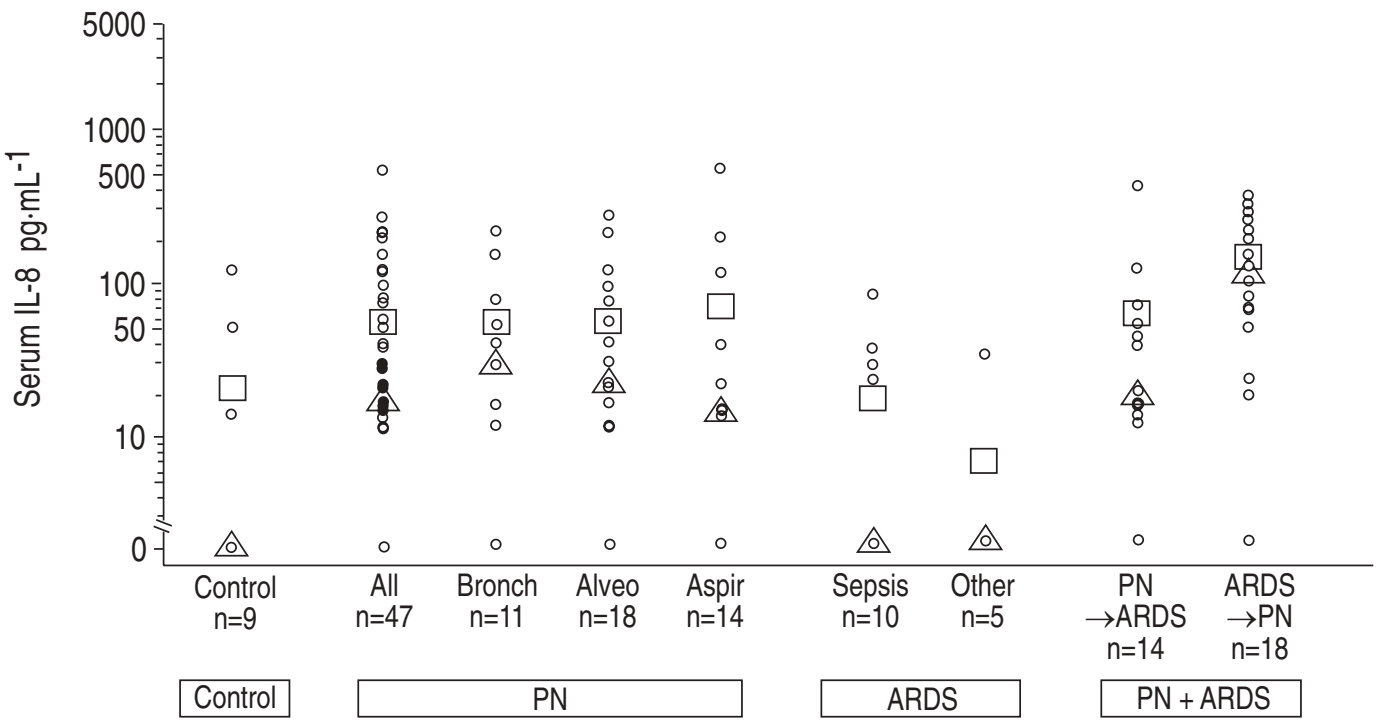

Fig. 6. - Serum IL-8 values in patients with pneumonia (PN), acute respiratory distress syndrome (ARDS), combined forms (PN+ARDS) and controls. Open circles $(O)$ represent single measurements; additionally, mean $(\square)$ and median $(\Delta)$ of each subgroup are given. Serum IL-8 was significantly higher in the PN+ARDS group as compared to PN $(\mathrm{p}<0.01)$, ARDS $(\mathrm{p}<0.001)$ and controls $(\mathrm{p}<0.01)$, whereas PN or ARDS were not different from control values. Subgroups within these main groups did not differ from each other, except for higher values in ARDS $\rightarrow$ PN patients as compared to $\mathrm{PN} \rightarrow \mathrm{ARDS}(\mathrm{p}<0.05)$. IL-8: interleukin-8. For further definitions see legend to figure 1 . 


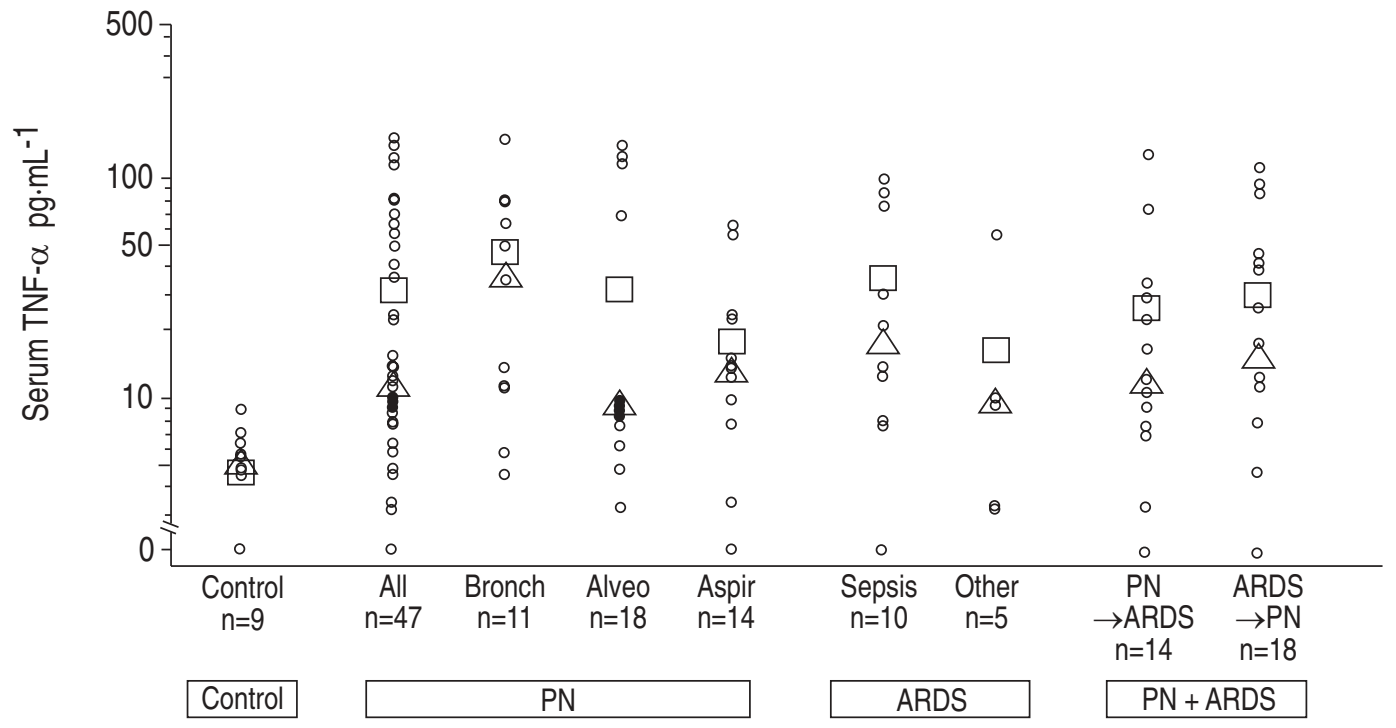

Fig. 7. - Serum TNF- $\alpha$ values in patients with pneumonia (PN), acute respiratory distress syndrome (ARDS), combined forms (PN+ARDS) and controls. Open circles $(O)$ represent single measurements; additionally, mean $(\square)$ and median $(\Delta)$ of each subgroup are given. PN, ARDS and PN+ARDS were all significantly different from controls $(\mathrm{p}<0.01, \mathrm{p}<0.001$ and $\mathrm{p}<0.05$, respectively). Differences between these main groups and between subgroups of either main group were not observed. TNF- $\alpha$ : tumour necrosis factor- $\alpha$. For further definitions see legend to figure 1.

Table 3. - Cytokine levels in patients with acute respiratory distress syndrome (ARDS), pneumonia (PN), or with a combination of ARDS and PN: comparison of survivors and nonsurvivors

\begin{tabular}{|c|c|c|c|c|c|c|}
\hline & \multicolumn{2}{|c|}{ ARDS } & \multicolumn{2}{|c|}{ PN } & \multicolumn{2}{|c|}{ PN + ARDS } \\
\hline & Survivors & $\begin{array}{c}\text { Non- } \\
\text { survivors }\end{array}$ & Survivors & $\begin{array}{c}\text { Non- } \\
\text { survivors }\end{array}$ & Survivors & $\begin{array}{c}\text { Non- } \\
\text { survivors }\end{array}$ \\
\hline BAL & $28.6 \pm 88$ & $212 \pm 83$ & $258 \pm 59$ & $328 \pm 87$ & $431 \pm 99$ & $323 \pm 142$ \\
\hline $\mathrm{IL}-8 \mathrm{pg} \cdot \mathrm{mL}^{-1}$ & $(244.2)$ & $(180.8)$ & (189.6) & $(172.5)$ & $(352.9)$ & (153) \\
\hline Serum & $6.1 \pm 3.8$ & $17 \pm 11$ & $34 \pm 12$ & $68 \pm 20^{*}$ & $110 \pm 35$ & $40 \pm 16$ \\
\hline $\mathrm{IL}-8 \mathrm{pg} \cdot \mathrm{mL}^{-1}$ & (0) & (0) & $(13.2)$ & (28) & $(96.5)$ & $(31.1)$ \\
\hline BAL & $3772 \pm 2492$ & $705 \pm 572$ & $5437 \pm 4078$ & $1533 \pm 824$ & $1448 \pm 567$ & $148 \pm 29$ \\
\hline $\mathrm{IL}-6 \mathrm{pg} \cdot \mathrm{mL}^{-1}$ & (1780) & (31) & (186) & $(298)$ & (280) & (174) \\
\hline Serum & $275 \pm 129$ & $600 \pm 240$ & $889 \pm 432$ & $2907 \pm 2438$ & $1785 \pm 659$ & $568 \pm 317$ \\
\hline IL-6 $\mathrm{pg} \cdot \mathrm{mL}^{-1}$ & (96) & (252) & (281) & (306) & (366) & (202) \\
\hline Serum & $31.6 \pm 15.4$ & $31.7 \pm 13.3$ & $18.3 \pm 4.2$ & $51.3 \pm 12.7$ & $38.2 \pm 11.5$ & $19.2 \pm 4.3$ \\
\hline $\mathrm{TNF}-\alpha \quad \mathrm{pg} \cdot \mathrm{mL}^{-1}$ & (14.2) & (10.5) & (11.5) & (37) & $(28.6)$ & (18.6) \\
\hline
\end{tabular}

BAL values are presented as directly measured concentrations without urea-correction for dilution. Values are presented as mean \pm SEM and median in brackets for each group. If more than one examination was performed in one single patient, mean values for each single patients were considered. Significant differences between survivors and nonsurvivors were not observed for any cytokine (Wilcoxon-Mann-Whitney test), except for higher serum IL-8 in nonsurviving PN patients (*: p<0.05). IL-8: interleukin-8; IL-6: interleukin-6; TNF- $\alpha$ : tumour necrosis factor- $\alpha$; BAL: bronchoalveolar lavage.

lung injury (ALI) and ARDS include: timing (acute onset); oxygenation $\left(\mathrm{Pa}, \mathrm{O}_{2} / F \mathrm{I}, \mathrm{O}_{2}<300 \mathrm{mmHg}\right.$ in ALI and $<200$ $\mathrm{mmHg}$ in ARDS); radiographic signs (bilateral infiltrates) and a capillary wedge pressure $<18 \mathrm{mmHg}$. There was controversy regarding diffuse lung infection: "The question is, if a pulmonary infection becomes diffuse and severe enough to meet ARDS criteria, should it be included under the ALI/ARDS definitions?" [31]. The consensus was that severe pulmonary infection should be considered ALI/ARDS when the physiological criteria listed above are met, although "this was not a unanimous decision".

The current study focused on the question, of whether primary or secondary lung infection provokes cytokine profiles that differ from ARDS primarily caused by extrapulmonary factors. This issue is worthy of study, as severe PN is assumed to be an increasingly important antecedent of severe respiratory failure [35]; it was, in fact, the quantitatively predominant underlying disease demanding mechanical ventilation in this patient group studied over 2 yrs. Moreover, secondary pneumonia acquired during the course of ARDS, although often difficult to diagnose, has been shown to aggravate the disease and to reduce survival rate [36-38]. For the purpose of this study, ARDS primarily caused by extrapulmonary factors (mostly sepsis of nonpulmonary origin) was, thus, separated from respiratory failure due to severe PN. A third group included patients presenting characteristics of both categories: ARDS $\rightarrow \mathrm{PN}$ were patients that acquired microbiologically proven pneumonia in the course of ARDS, and PN $\rightarrow$ ARDS reflected diffuse spreading of lung infiltrates in patients initially presenting with circumscript (microbiologically classified) pneumonia. In addition, patients with cardiogenic pulmonary 
Table 4. - Correlations between IL-6 and IL-8 concentrations and selected basic variables in patients with pneumonia (PN), acute respiratory distress syndrome (ARDS) and combined forms (PN+ARDS)

\begin{tabular}{lccc}
\hline & PN & ARDS & PN + ARDS \\
\hline IL-8 BAL & 0.164 & 0.6929 & 0.3793 \\
Protein BAL & NS & $\mathrm{p}<0.01$ & $\mathrm{p}<0.05$ \\
IL-8 BAL & 0.5427 & 0.3879 & 0.0522 \\
\% PMN BAL & $\mathrm{p}<0.001$ & $\mathrm{NS}$ & $\mathrm{NS}$ \\
IL-8 BAL & 0.5054 & 0.5549 & 0.0753 \\
PMN counts BAL & $\mathrm{p}<0.001$ & $\mathrm{p}<0.059$ & $\mathrm{NS}$ \\
IL-6 BAL & 0.5807 & 0.3324 & 0.5825 \\
Protein BAL & $\mathrm{p}<0.001$ & $\mathrm{NS}$ & $\mathrm{p}<0.001$ \\
IL-6 BAL & 0.5757 & 0.58 & 0.1613 \\
\% PMN BAL & $\mathrm{p}<0.001$ & $\mathrm{P}<0.05$ & $\mathrm{NS}$ \\
IL-6 BAL & 0.5088 & 0.5805 & 0.2078 \\
PMN counts BAL & $\mathrm{p}<0.001$ & $\mathrm{p}<0.05$ & $\mathrm{NS}$ \\
IL-6 serum & 0.4393 & 0.4196 & 0.5918 \\
CRP & $\mathrm{p}<0.01$ & $\mathrm{NS}$ & $\mathrm{p}<0.001$ \\
\hline
\end{tabular}

Nonparametric Spearman's correlation coefficients are given. For calculations, protein and cytokine BAL values were considered as directly measured concentrations without urea-correction for dilution. NS: not significant; PMN: polymorphonuclear neutrophils. CRP: c-reactive protein. For further definitions see legend to table 2 .

Table 5. - Correlations between IL-6 and IL-8 concentrations in BAL fluids and serum samples in patients with pneumonia (PN), acute respiratory distress syndrome (ARDS) and combined forms (PN+ARDS)

\begin{tabular}{lccc}
\hline & PN & ARDS & PN + ARDS \\
\hline IL-8 BAL & 0.2745 & -0.2658 & 0.2138 \\
IL-8 serum & NS & NS & NS \\
IL-8 BAL & 0.2243 & 0.1966 & 0.1527 \\
IL-6 serum & NS & NS & NS \\
IL-8 BAL & 0.4558 & 0.575 & 0.3679 \\
IL-6 BAL & $\mathrm{p}<0.001$ & $\mathrm{p}<0.05$ & $\mathrm{p}<0.05$ \\
IL-6 BAL & 0.3066 & 0.0191 & 0.3853 \\
IL-8 serum & $\mathrm{p}<0.05$ & NS & $\mathrm{p}<0.05$ \\
IL-6 BAL & 0.5423 & 0.3324 & 0.6793 \\
IL-6 serum & $\mathrm{p}<0.001$ & NS & $\mathrm{p}<0.001$ \\
IL-6 serum & 0.2567 & 0.356 & 0.468 \\
IL-8 serum & NS & NS & $\mathrm{p}<0.01$ \\
\hline
\end{tabular}

Nonparametric Spearman's correlation coefficients are given. For calculations, cytokine BAL values were considered as directly measured concentrations without urea-correction for dilution. NS: not significant. For further definitions see legend to table 2 .

oedema were included as a further "control" group, to probe the impact of functional lung disturbances on cytokine changes in the absence of primary inflammatory disease; the gas exchange abnormalities in these patients matched those with ARDS and/or severe PN very well, but were restricted to the first $48 \mathrm{~h}$ after onset of mechanical ventilation.

\section{Interleukin-8}

BAL IL-8 levels were markedly elevated in virtually all patients with ARDS and/or PN, and this was true for the early phase of disease as well as later time periods
(>10 days). Alveolar space IL-8 concentrations were substantially higher than circulating IL-8 levels, which is in accordance with predominant local generation of this cytokine. The data on BAL IL-8 support previous studies demonstrating consistently increased lavage IL-8 concentrations in ARDS patients [3, 4, 39], and in patients at risk for this disease [2]. This inflammatory cytokine allowed a clear separation of ARDS/PN from cardiogenic pulmonary oedema, in which only moderately elevated levels were noted; previous data for the latter group are not known to the authors.

In contrast, BAL IL-8 levels did not discriminate between ARDS and primary or secondary lung infection, and no discrimination within the subgroups of pneumonia was achieved. This result differs somewhat from investigations by ChOllet-Martin et al. [1], in which higher lavage IL-8 levels were noted in ARDS states as compared to a limited number $(n=6)$ of PN patients. However, this difference may be explained by the fact that, in their study, the ARDS patients displayed more severe gas exchange disturbances than the PN patients, whereas the degree of functional impairment was comparable in the current investigation. From experimental data, IL-8 is known to be an important chemoattractant effecting neutrophil recruitment into the alveolar space $[40,41]$. In clinical studies in ARDS patients, a significant correlation between BAL IL-8 levels and alveolar neutrophil numbers was occasionally found [3, 39], but was also missing in some cases [1]. In the current study, such correlation was evident in patients with ARDS and $\mathrm{PN}$, but - for presently unknown reasons - not for the combined group (PN + ARDS).

As anticipated from the previously described features of IL-8, elevations of serum concentrations of this cytokine were less marked than the rise in the levels in the alveolar space in all groups of patients [1, 4]. This finding does not refute the hypothesis that under conditions of a predominance of sepsis and septic shock, substantial IL-8 concentrations may appear in the vascular compartment $[5,7,42]$. Although the BAL IL-8 levels were greatly increased in all patients with ARDS and/or $\mathrm{PN}$, no association was observed between the level of this cytokine and survival rate, either in the ARDS, the PN or the combined (PN + ARDS) group. Such a predictive value of BAL IL-8 for mortality has been suggested from preceding studies $[1,3]$.

\section{Interleukin-6}

IL-6 is known to appear in the vascular compartment in large quantities under conditions of sepsis and septic shock [5, 17, 18, 22, 25, 26]. However, its pathogenic significance as being a cause or a consequence in these disease states is not exactly known. Persistence of elevated serum IL-6 concentrations was described as a characteristic indicator for the development of multiplesystem organ failure [22], and high IL-6 levels were found to be associated with a poor prognosis in septic shock $[5,25,26]$. We found highly elevated IL-6 concentrations circulating in all patients with ARDS, and similarly in PN, in contrast to patients with cardiogenic pulmonary oedema. This finding suggests that the systemic inflammatory response was comparable under conditions of ARDS - where it was anticipated - and in states 
of severe PN requiring mechanical ventilation. In accordance with this interpretation, the serum CRP levels in PN were only slightly lower than those in ARDS. In contrast to the previous reports mentioned above $[5,6,22$, 26], we again observed no significant differences between survivors and nonsurvivors.

Interestingly, in patients with ARDS and/or PN IL-6 levels in BAL and serum were similarly elevated. Given the dilution by the lavage fluid the level in the alveolar space must have been markedly greater than in the intravascular space. This finding again signals the predominance of local production, as discussed for IL-8. Interestingly, the correlations between BAL cytokine levels and neutrophil influx, were of similar strength for IL-6 and IL-8. These findings may suggest a local proinflammatory role for this "alarm hormone", but the data supporting such a view are much more sparse than those related to IL-8 [27-30].

\section{Tumour necrosis factor}

In previous investigations, elevated alveolar space TNF$\alpha$ levels were found in patients with ARDS [19, 20, 24]. In contrast, TNF- $\alpha$ was very rarely detected in the BAL fluids either in ARDS or in severe PN in the current study. As this result was puzzling, an attempt was made to exclude any methodological pitfalls: two different immunological tests were employed and the samples were analysed in a bioassay system previously reported by our laboratory to be suitable to quantify TNF- $\alpha$ generated under experimental conditions [43, 44]. Moreover, excellent recovery of recombinant TNF- $\alpha$ was demonstrated when mixed to BAL fluids immediately after performing the lavage procedure, thereby excluding the possibility that TNF- $\alpha$ vanishes due to processing, transport or storage procedures. Thus, the present finding of a near total absence of TNF- $\alpha$ in BAL fluids is wellfounded. PARSONS et al. [21] explained discrepancies in TNF- $\alpha$ determinations by differences in the timing of measurement, and this may, indeed, be an important factor with respect to the short half-time of this cytokine (14-18 min [45]). This view has been confirmed by other investigators, who found elevated TNF- $\alpha$ mainly - but not in all cases - within the early phase after onset of disease $[19,24]$. We may, thus, have missed early peaks of TNF- $\alpha$ generation due to our lavage protocol dictated by clinical necessities. Dilution of the alveolar space concentrations by the lavage fluid may be another important factor to explain the discrepancy between the current and preceding studies, in which TNF- $\alpha$ was directly quantified in bronchoalveolar aspirates [20, 24].

In contrast to the analysis of BAL fluids, elevated levels of circulating TNF- $\alpha$ were observed in the majority of the patients with ARDS and/or PN, which is in accordance with previous observations [18, 19, 23, 46]. Again, there was no difference between the main categories of ARDS and pneumonia, and among different subgroups of pneumonia. These findings further support the view of a major systemic inflammatory response in all of these patients, as suggested previously [47].

Alveolar space cytokine sampling by lavage demonstrated rapid onset and persistent local generation of interleukin- 8 and interleukin-6 in patients with acute res- piratory distress syndrome and severe pneumonia. This profile clearly separated those patients with inflammatory lung injury from healthy controls and from patients with comparable functional impairment due to cardiogenic pulmonary oedema. However, the alveolar space cytokines, interleukin- 8 and interleukin- 6 did not discriminate between acute respiratory distress syndrome in the absence of lung infection and states of severe primary or secondary pneumonia, as well as among different subgroups of pneumonia. In addition, circulating interleukin- 6 and tumour necrosis factor- $\alpha$ and - to lesser extent - interleukin-8 levels suggested systemic inflammatory responses in association with the lung infection, that were again clearly separate from cardiogenic oedema, but comparable for the various entities of acute respiratory distress syndrome and/or severe pneumonia. No single variable (alveolar or intravascular cytokine) was predictive for survival. In general, these findings support similarities in local and systemic inflammatory processes between acute respiratory distress syndrome in the absence of lung infection and states of severe pneumonia.

\section{References}

1. Chollet-Martin S, Montravers P, Gibert C, et al. High levels of interleukin-8 in the blood and alveolar spaces of patients with pneumonia and adult respiratory distress syndrome. Infect Immun 1993; 61: 4553-4559.

2. Donnelly SC, Strieter RM, Kunkel SL, et al. Interleukin8 and development of adult respiratory distress syndrome in at-risk patient groups. Lancet 1993; 341: 643-647.

3. Miller EJ, Cohen AB, Nagao S, et al. Elevated levels of NAP-1/interleukin- 8 are present in the airspaces of patients with the adult respiratory distress syndrome and are associated with increased mortality. Am Rev Respir Dis 1992; 148: 427-432.

4. Torre D, Zeroli C, Giola M, et al. Levels of interleukin8 in patients with adult respiratory distress syndrome. $J$ Infect Dis 1993; 167: 505-506.

5. Friedland JS, Supputtamongkol Y, Remick DG, et al. Prolonged elevation of interleukin- 8 and interleukin-6 concentrations in plasma and of leukocyte interleukin-8 mRNA levels during septicemic and localized Pseudomans pseudomallei infection. Infect Immun 1992; 60: 24022408.

6. Hack CE, Hart M, Strack van Schijndel RJM, et al. Interleukin-8 in sepsis: relation to shock and inflammatory mediators. Infect Immun 1992; 60: 2835-2842.

7. Marty C, Misset B, Tamion F, et al. Circulating interleukin-8 concentrations in patients with multiple organ failure of septic and nonseptic origin. Crit Care Med 1994; 22: 673.

8. Kelley J. Cytokines of the lung. Am Rev Respir Dis 1990; 141: 765-788.

9. Manogue KR, van Deventer SJH, Cerami A. Tumor necrosis factor-alpha or cachectin. The Cytokine Handbook. Chap. 12. New York, Academic Press Ltd, 1991; pp. 241-256.

10. Cannon JG, Tompkins RG, Gelfand JA, et al. Circulating interleukin-1 and tumor necrosis factor in septic shock and experimental endotoxin fever. $J$ Infect Dis 1990; 161: 79-84.

11. Cerami A, Beutler B. The role of cachectin/TNF in endotoxic shock and cachexia. Immunol Today 1988; 9: 28-31. 
12. van Deventer SJH, Büller HR, ten Cate JW, et al. Experimental endotoxemia in humans: analysis of cytokine release and coagulation fibrinolytic, and complement pathways. Blood 1990; 76: 2520-2526.

13. Tracey KJ, Fong Y, Hesse DG, et al. Anti-cachectin/TNF monoclonal antibodies prevent septic shock during lethal bacteraemia. Nature 1987; 330: 662-664.

14. Leeper-Woodford SK, Carey PD, Byrne K, et al. Tumor necrosis factor - alpha and beta subtypes appear in circulation during onset of sepsis-induced lung injury. Am Rev Respir Dis 1991; 143: 1076-1082.

15. Tracey KJ, Beutler B, Lowry SF, et al. Shock and tissue injury induced by recombinant human cachectin. Science 1986; 234: 470-474.

16. Debets JMH, Kampneijer R, van der Linden MPMH, et al. Plasma tumor necrosis factor and mortality in critically ill septic patients. Crit Care Med 1989; 17: 489_ 494.

17. Dofferhoff ASM, Bom VJJ, de Vries-Hospers HG, et al. Patterns of cytokines, plasma endotoxin, plasminogen activator inhibitor, and acute-phase proteins during the treatment of severe sepsis in humans. Crit Care Med 1992; 20: 185-192.

18. Donnelly TJ, Meade P, Jagels M, et al. Cytokine, complement and endotoxin profiles associated with the development of the adult respiratory distress syndrome after severe injury. Crit Care Med 1994; 22: 768-776.

19. Hyers TM, Tricomi SM, Dettenmeyer PA, et al. Tumor necrosis factor levels in serum and bronchoalveolar lavage fluid of patients with the adult respiratory distress syndrome. Am Rev Respir Dis 1991; 144: 266-271.

20. Millar AB, Fowley NM, Singer M, et al. Tumor necrosis factor in bronchopulmonary secretions of patients with adult respiratory distress syndrome. Lancet 1989; 23: 712-714.

21. Parsons PE, Moore FA, Moore EE, et al. Studies on the role of tumor necrosis factor in adult respiratory distress syndrome. Am Rev Respir Dis 1992; 146: 694-700.

22. Pinsky MR, Vincent J-L, Deviere J, et al. Serum cytokine levels in human septic shock: relation to multiple-system organ failure and mortality. Chest 1993; 103: 565575.

23. Roten R, Markert M, Feihl F, et al. Plasma levels of tumor necrosis factor in the adult respiratory distress syndrome. Am Rev Respir Dis 1991; 43: 590-592.

24. Suter PM, Suter S, Girardin E, et al. High bronchoalveolar levels of tumor necrosis factor and its inhibitors, interleukin-1, interferon and elastase, in patients with adult respiratory distress syndrome after trauma, shock or sepsis. Am Rev Respir Dis 1992; 145: 1016-1022.

25. Hack CE, De Groot ER, Felt-Bersma JF, et al. Increased plasma levels of interleukin-6 in sepsis. Blood 1989; 74: 1704-1710.

26. Waage A, Brandtzaeg P, Halstensen A, et al. The complex pattern of cytokines in serum from patients with meningococcal septic shock. J Exp Med 1989; 169: 333-338

27. Grigg JM, Barber A, Silverman M. Increased levels of bronchoalveolar lavage fluid interleukin-6 in preterm ventilated infants after prolonged rupture of membranes. $A m$ Rev Respir Dis 1992; 145: 782-786.

28. Humbert M, Delattre RM, Fattal S, et al. In situ production of interleukin-6 within human lung allografts displaying rejection or cytomegalovirus pneumonia. Transplantation 1993; 56: 623-627.

29. Lindsey HJ, Kisala JM, Ayala A, et al. Pentoxifylline attenuates oxygen-induced lung injury. J Surg Res 1994; 56: 543-548.

30. Trentin L, Garbisa S, Zambello R, et al. Spontaneous production of interleukin- 6 by alveolar macrophages from human immunodeficiency virus type-1-infected patients. J Infect Dis 1992; 166: 731.

31. Bernard GR, Artigas A, Brigham KL, et al. The AmericanEuropean Consensus Conference on ARDS. Am J Respir Crit Care Med 1994; 149: 818-824.

32. Fraser RG (Ed), Paré JAP, Paré PD, et al. Diagnosis of diseases of the chest. 3rd edn. Philadelphia, W.B. Saunders Co., 1989.

33. Rennard SI, Basset G, Lecossier D, et al. Estimation of volume of epithelial lining fluid recovered by lavage using urea as marker of dilution. J Appl Physiol 1986; 60: $532-538$

34. Espevik T, Nissen-Meyer J. A highly sensitive cell line, WEHI 164 clone 13, for measuring cytotoxic factor/tumor necrosis factor from human monocytes. $J$ Immunol Meth 1986; 95: 99.

35. Sloane PJ, Gee MH, Gottlieb JE, et al. A multicenter registry of patients with acute respiratory distress syndrome. Am Rev Respir Dis 1992; 146: 419-426.

36. Campbell GD, Coalson JJ, Johanson WG. The effect of bacterial superinfection on lung function after diffuse alveolar damage. Am Rev Respir Dis 1984; 129: 974978.

37. Hyers TM, Fowler AA. Adult respiratory distress syndrome: causes, morbidity and mortality. Fed Proc 1986; 45: $25-29$.

38. Seidenfeld JJ, Pohl DF, Bell RC, et al. Incidence, site and outcome of infections in patients with the adult respiratory distress syndrome. Am Rev Respir Dis 1986; 134: $12-16$.

39. Jorens PG, Van Damme J, De Backer W, et al. Interleukin 8 (IL-8) in the bronchoalveolar lavage fluid from patients with the adult respiratory distress syndrome (ARDS) and patients at risk for ARDS. Cytokine 1992; 4: 592-597.

40. Kunkel SL, Standiford T, Kasahara K, et al. Interleukin8 (IL-8): the major neutrophil chemotactic factor in the lung. Exp Lung Res 1991; 17: 17-23.

41. Van Damme J. Interleukin-8 and related molecules. In: Thomson AW, ed. The Cytokine Handbook: Immunology and Molecular Biology of Cytokines. New York, Academic Press, 1991; pp. 201-214.

42. Van Zee KJ, DeForge LE, Fischer E, et al. IL-8 in septic shock, endotoxemia, and after IL-1 administration. $J$ Immunol 1991; 146: 3478-3482.

43. Walmrath D, Ghofrani AH, Rosseau S, et al. Endotoxin "priming" potentiates lung responsiveness to E. coli hemolysin: an example of endo- and exotoxin co-operativity. J Exp Med 1994; 180: 1437-1443.

44. Walmrath D, Griebner M, Kolb B, et al. Endotoxin primes perfused rabbit lungs for enhanced vasoconstrictor response to staphylococcal $\alpha$-toxin. Am Rev Respir Dis 1993; 148: 1179-1186.

45. Blick M, Sherwin SA, Rosenblum M, et al. Phase I study of recombinant tumor necrosis factor in cancer patients. Cancer Res 1987; 47: 2986-2989.

46. Chollet-Martin S, Montravers P, Gibert C, et al. Subpopulation of hyperresponsive polymorphonuclear neutrophils in patients with adult respiratory distress syndrome. Am Rev Respir Dis 1992; 146: 990-996.

47. Meduri GU, Headley S, Kohler G, et al. Persistent elevation of inflammatory cytokines predicts a poor outcome in ARDS. Chest 1995; 107: 1062-1173. 